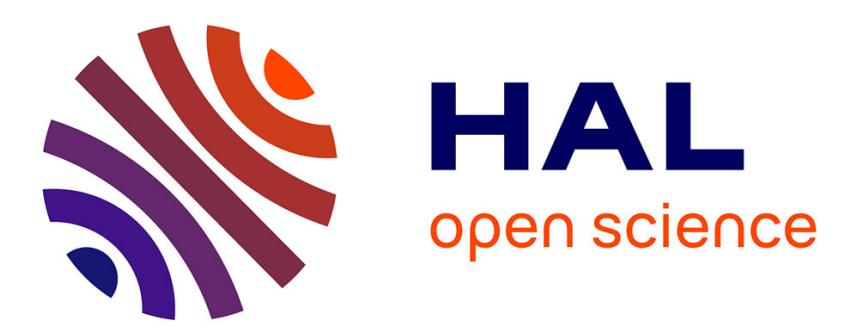

\title{
Expansion in series of exponential polynomials of mean-periodic functions
}

Habib Ouerdiane, Myriam Ounaïes

\section{To cite this version:}

Habib Ouerdiane, Myriam Ounaïes. Expansion in series of exponential polynomials of mean-periodic functions. Complex Variables and Elliptic Equations, 2012, 10.1080/17476933.2010.487204 . hal01289864

\section{HAL Id: hal-01289864 \\ https://hal.science/hal-01289864}

Submitted on 17 Mar 2016

HAL is a multi-disciplinary open access archive for the deposit and dissemination of scientific research documents, whether they are published or not. The documents may come from teaching and research institutions in France or abroad, or from public or private research centers.
L'archive ouverte pluridisciplinaire $\mathbf{H A L}$, est destinée au dépôt et à la diffusion de documents scientifiques de niveau recherche, publiés ou non, émanant des établissements d'enseignement et de recherche français ou étrangers, des laboratoires publics ou privés. 


\title{
EXPANSION IN SERIES OF EXPONENTIAL POLYNOMIALS OF MEAN-PERIODIC FUNCTIONS
}

\author{
H. OUERDIANE AND M. OUNAÏES
}

\begin{abstract}
Let $\theta$ be a Young function and consider the space $\mathcal{F}_{\theta}(\mathbb{C})$ of all entire functions on $\mathbb{C}$ with $\theta$-exponential growth. In this paper, we are interested in the solutions $f \in \mathcal{F}_{\theta}(\mathbb{C})$ of the convolution equation $T \star f=0$, called mean-periodic functions, where $T$ is in the topological dual of $\mathcal{F}_{\theta}(\mathbb{C})$. We show that each mean-periodic function admits an expansion as a convergent series of exponential polynomials.
\end{abstract}

\section{INTRODUCTION.}

A periodic function $f$ with period $t$ may be defined in terms of convolution equation as a function satisfying the convolution equation

$$
\left(\delta_{t}-\delta_{0}\right) \star f=0,
$$

while a function with zero average over an interval of length $t>0$ satisfies the convolution equation

$$
\mu \star f=0,
$$

where $\mu$ is defined by $<\mu, f>=\frac{1}{t} \int_{-t / 2}^{t / 2} f(x) d x$. From the observation that the second notion is more natural from the point of view of experimental physics, Delsartes generalized the concept of periodic functions by introducing in [7] the notion of "mean-periodic" functions as the solutions of homogeneous convolution equations.

In this paper, we are dealing with the problem of expressing mean periodic functions as series of exponentials polynomials.

Let us denote by $\mathcal{H}(\mathbb{C})$ the space of all entire functions on $\mathbb{C}$. Let $\theta:[0,+\infty[\rightarrow[0,+\infty[$ be a Young function that is, a convex, continous and increasing function, vanishing at the origin and such that $t=o(\theta(t))$. Let $\theta^{*}$ be its Legendre transform, which is also a Young function. Denote by $\mathcal{F}_{\theta}(\mathbb{C})$ the space of all functions $f \in \mathcal{H}(\mathbb{C})$ such that, for all constants $m>0$,

$$
\sup _{z \in \mathbb{C}}|f(z)| e^{-\theta^{*}(m|z|)} \cdot<\infty
$$

endowed with the projective limit topology.

Examples of Young functions are

Date: May 21, 2010.

1991 Mathematics Subject Classification. 30D15, 41A05, 46E10, 44A35.

Key words and phrases. entire functions, convolution equations, mean-periodic functions, interpolating sequences.

*Corresponding author. Email: ounaies@math.u-strasbg.fr. 
- $\theta(t)=t^{a}, a>1$

- $\theta(t)=t \ln t-t$

- $\theta(t)=e^{t}-1$.

We will also consider the limit case where $\theta(t)=t$. In this case, the associated conjugate function $\theta^{*}$ is formally infinite. Therefore, no growth condition of the type (1) is involved and we put $\mathcal{F}_{\theta}(\mathbb{C})=\mathcal{H}(\mathbb{C})$ endowed with the topology of uniform convergence on compact sets.

It follows from the definitions that the spaces $\mathcal{F}_{\theta}(\mathbb{C})$ are invariant under translation and contain all exponential monomials $z^{n} e^{\alpha z}, n \in \mathbb{N}, \alpha \in \mathbb{C}$.

We will say that $f \in \mathcal{F}_{\theta}(\mathbb{C})$ is a mean-periodic function if, for a certain non zero analytic functional $T \in \mathcal{F}_{\theta}^{\prime}(\mathbb{C}), f$ verifies the convolution equation

$$
T \star f=0 .
$$

In this case, we say that $f$ is $T$-mean-periodic.

If we denote by $\left\{\alpha_{k}\right\}_{k}$ the zeros of the Fourier-Borel transform of $T$ and $m_{k}$ their order of multiplicity, then all exponential monomials $z^{j} e^{\alpha_{k} z}$, with $j<m_{k}$ are $T$-mean-periodic functions (see Lemma 5). Theses exponential monomials are called the elementary solutions of (2).

Let us note that the kernel of $T \star$ is a closed subspace of $\mathcal{F}_{\theta}(\mathbb{C})$ which is invariant under translation.

Our work is related to the classical spectral synthesis problem, that is, the one of finding conditions under which a closed subspace $S \in \mathcal{F}_{\theta}(\mathbb{C})$ which is invariant under translation coïncides with the closure of the span of the exponential monomials contained in $S$. When this is the case, $S$ is said to admit spectral synthesis. It is a famous result of L. Schwartz ([18]) that when we're dealing with $\mathcal{H}(\mathbb{C})$, every non zero closed invariant subspace admits spectral synthesis.

The problem of spectral synthesis for a large class of spaces of entire functions with growth conditions is dealt with in the works by I.F. Krasičkov ([11, 12, 13]). These spaces have in common with our setting the cases $\theta(t)=t^{a}$.

The fundamental principal problem concerns closed invariant subspaces admitting spectral synthesis, in particular, when dealing with the kernel of $T \star$, it asks whether every solution to the equation (2) can be represented in terms of elementary solutions as an absolutely convergent series in $\mathcal{F}_{\theta}(\mathbb{C})$ of the form

$$
\sum_{k} e^{z \alpha_{k}} \sum_{l=0}^{m_{k}-1} c_{k, l} z^{l} .
$$

This question is closely connected to the theory of interpolation in spaces of entire functions (see [2],[6]).

We will use the interpolation result obtained in [16] to obtain an explicit expansion of every function in the kernel of $T \star$ in terms of the elementary solutions (see Theorem 6), using an Abel summation procedure in order to make the sum convergent. In fact, we prove that any $T$-mean-periodic function $f \in \mathcal{F}_{\theta}(\mathbb{C})$ admits the following expansion as a convergent series in $\mathcal{F}_{\theta}(\mathbb{C})$

$$
f(z)=\sum_{k} \sum_{l=0}^{m_{k}-1} c_{k, l}\left[\sum_{j=1}^{k} e^{z \alpha_{j}} P_{k, j, l}(z)\right]
$$


where $P_{k, j, l}$ are polynomials of degree $<m_{j}$, explicitely given by (23) and (24) in terms of $\left\{\left(\alpha_{k}, m_{k}\right)\right\}_{k}$. Moreover, the coefficients $c_{k, l}$ verify the growth condition $(10)$ and can be explicitly computed in terms of $f$ and $T$.

When $V=\left\{\left(\alpha_{k}, m_{k}\right)\right\}_{k}$ is an interpolating variety (see Definition 5), we don't need the Abellike summation, we simply obtain that any $T$-mean-periodic function $f \in \mathcal{F}_{\theta}(\mathbb{C})$ admits the following expansion as a convergent series in $\mathcal{F}_{\theta}(\mathbb{C})$

$$
f(z)=\sum_{k} e^{z \alpha_{k}} \sum_{l=0}^{m_{k}-1} d_{k, l} \frac{z^{l}}{l !},
$$

where the coefficients $d_{k, l}$ verify the growth estimate (26) (see Theorem 12).

These results generalize those obtained in [2] and [6], where the authors considered the case $\mathcal{F}_{\theta}(\mathbb{C})=\mathcal{H}(\mathbb{C})$. In fact, they showed that, given $T \in \mathcal{H}^{\prime}(\mathbb{C})$ ), there exists a sequence of indices $k_{1}=1<k_{2}<\cdots$ such that any $T$-mean periodic function $f \in \mathcal{H}(\mathbb{C})$ admits a unique expansion, convergent in $\mathcal{H}(\mathbb{C})$, of the form

$$
f(z)=\sum_{n \geq 1} \sum_{k_{n} \leq k<k_{n+1}} e^{\alpha_{k} z} \sum_{j=0}^{m_{k}-1} d_{k, j} \frac{z^{j}}{j !} .
$$

In (5), the sum converges by grouping the terms and not by an Abel process, but in general, the sequence $\left\{k_{n}\right\}_{n}$ is not explicit, except in the case when $V$ is an interpolating variety, where the sequence $k_{n}=n$ works, thus formula (5) leads to (4).

The paper is organized as follows : Section 2 is devoted to preliminary definitions and useful results from functional analysis. The main results are stated in Section 3 and the proofs are given in Section 4. Finally, in Section 5, we study the particular case when $V$ is an interpolating variety.

\section{PRELIMINARIES AND DEFINITIONS.}

Definition. A function $\theta:[0,+\infty[\rightarrow[0,+\infty[$ is called a Young function if it is convex, continuous, increasing and verifies $\theta(0)=0$ and $r=o(\theta(r))$ when $r \rightarrow+\infty$.

Definition. Let $\theta$ be a Young function. The Legendre transform $\theta^{*}$ of $\theta$ is the function defined by

$$
\theta^{*}(x)=\sup _{t \geq 0}(t x-\theta(t))
$$

Note that the Legendre transform of a Young function is a Young function and $\theta^{* *}=\theta$. We refer the reader to [14] for further details about Young functions.

Throughout the paper, $\theta$ will denote either the function $\theta(x)=x$ or a Young function.

For any $m>0$, consider $E_{\theta, m}(\mathbb{C})$, the Banach space of all functions $f \in \mathcal{H}(\mathbb{C})$ such that

$$
\|f\|_{\theta, m}:=\sup _{z \in \mathbb{C}}|f(z)| e^{-\theta(m|z|)}<+\infty
$$

and define

$$
\mathcal{G}_{\theta}(\mathbb{C})=\cup_{p \in \mathbb{N}^{*}} E_{\theta, p}(\mathbb{C})
$$

endowed with the inductive limit topology. It is clear that $\mathcal{G}_{\theta}(\mathbb{C})$ is an algebra under the ordinary multiplication of functions. 
Remark 1. When $\theta(x)=x^{k}, k \geq 1, \mathcal{G}_{\theta}(\mathbb{C})$ is the space of all entire functions, either of order $<k$ or of order $k$ and finite type. In particular, when $k=1, \mathcal{G}_{\theta}(\mathbb{C})$ is the space of all entire functions of exponential type, usually denoted by $\operatorname{Exp}(\mathbb{C})$.

We define the space $\mathcal{F}_{\theta}(\mathbb{C})$ as follows :

(i) In the case where $\theta(x)=x$, we put $\mathcal{F}_{\theta}(\mathbb{C})=\mathcal{H}(\mathbb{C})$, the space of all entire functions endowed with the topology of uniform convergence on every compact of $\mathbb{C}$. It is a FréchetSchwartz space (see [2]).

(ii) In the case where $\theta$ is a Young function, we denote

$$
\mathcal{F}_{\theta}(\mathbb{C})=\cap_{p \in \mathbb{N}^{*}} E_{\theta^{*}, 1 / p}(\mathbb{C})
$$

endowed with the projective limit topology. The space $\mathcal{F}_{\theta}(\mathbb{C})$ is a nuclear Fréchet space (see [9]), hence it is a Fréchet-Schwartz space (see [17]).

For any fixed $\xi \in \mathbb{C}$, and $l \in \mathbb{N}$, we will denote by $M_{l, \xi}$ the exponential monomial $z \rightarrow z^{l} e^{\xi z}$. It is easy to see that $M_{l, \xi} \in \mathcal{F}_{\theta}(\mathbb{C})$. In the next we denote by $\mathcal{F}_{\theta}^{\prime}(\mathbb{C})$ the strong topological dual of $\mathcal{F}_{\theta}(\mathbb{C})$.

Let us recall some definitions and properties from functional analysis. We refer to [2] for further details in the case (i) and to [9] for the case (ii).

To any fixed $u \in \mathbb{C}$, define the translation operator $\tau_{u}$ on $\mathcal{F}_{\theta}(\mathbb{C})$ by

$$
\left(\tau_{u} f\right)(z)=f(z+u), \text { for all } f \in \mathcal{F}_{\theta}(\mathbb{C}) \text { and } z \in \mathbb{C} .
$$

It's easy to see that $\mathcal{F}_{\theta}(\mathbb{C})$ is invariant under these translation operators.

For all $S \in \mathcal{F}_{\theta}^{\prime}(\mathbb{C})$ and $f \in \mathcal{F}_{\theta}(\mathbb{C})$, the function $z \rightarrow<S, \tau_{z} f>$, where $<,>$ denotes the duality bracket, is an element of $\mathcal{F}_{\theta}(\mathbb{C})$. Therefore, for any $S \in \mathcal{F}_{\theta}^{\prime}(\mathbb{C})$, the map $S \star: \mathcal{F}_{\theta}(\mathbb{C}) \rightarrow$ $\mathcal{F}_{\theta}(\mathbb{C})$ defined by

$$
S \star f(z)=<S, \tau_{z} f>
$$

is a convolution operator, i.e., it is linear, continuous and commute with any translation operator.

For any $S \in \mathcal{F}_{\theta}^{\prime}(\mathbb{C})$, the Fourier-Borel transform of $S$, denoted by $\mathcal{L}(S)$ is defined by

$$
\mathcal{L}(S)(\xi)=<S, e^{\xi \cdot}>
$$

where $e^{\xi \cdot}=M_{0, \xi}$ is the function $z \in \mathbb{C} \rightarrow e^{\xi z}$.

For any two elements $S$ and $U$ of $\mathcal{F}_{\theta}^{\prime}(\mathbb{C})$, the convolution product $S \star U \in \mathcal{F}_{\theta}^{\prime}(\mathbb{C})$ is defined by

$$
\forall f \in \mathcal{F}_{\theta}(\mathbb{C}), \quad<S \star U, f>=<S, U \star f>.
$$

Moreover for any $S, U \in \mathcal{F}_{\theta}^{\prime}(\mathbb{C})$

$$
\mathcal{L}(S \star U)=\mathcal{L}(S) \mathcal{L}(U)
$$

Under this convolution, $\mathcal{F}_{\theta}^{\prime}(\mathbb{C})$ is a commutative algebra admitting $\delta_{0}$, the Dirac measure at the origin, as unit.

Proposition 2. The Fourier-Borel transform $\mathcal{L}$ is a topological isomorphism between the alge$\operatorname{bras} \mathcal{F}_{\theta}^{\prime}(\mathbb{C})$ and $\mathcal{G}_{\theta}(\mathbb{C})$. 


\section{MAIN RESUlTS.}

Throughout the rest of the paper, let $T$ be a fixed non-zero element of $\mathcal{F}_{\theta}^{\prime}(\mathbb{C})$. Our main goal in this section is to show that any function $f \in \mathcal{F}_{\theta}(\mathbb{C})$ satisfying the equation

$$
T \star f=0
$$

can be represented as convergent series of exponential-polynomials which are themselves solution of (6).

Definition. We say that a function $f \in \mathcal{F}_{\theta}(\mathbb{C})$ is "T-mean-periodic" if it satisfies the equation (6).

Denote by $\Phi$ the entire function in $\mathcal{G}_{\theta}(\mathbb{C})$ defined by $\Phi=\mathcal{L}(T)$. Before going further, let us show the following division property :

Lemma 3. Let $h \in \mathcal{H}(\mathbb{C})$ and $g \in \mathcal{G}_{\theta}(\mathbb{C})$. If $g$ is not identically zero and if $f=g h \in \mathcal{G}_{\theta}(\mathbb{C})$, then $h \in \mathcal{G}_{\theta}(\mathbb{C})$.

Proof. Up to a translation, we may assume that $g(0) \neq 0$. Let us apply the minimum modulus theorem and it's corollary given in [2, Lemma 2.2.11] to the function $g$ in the disc of center 0 and radius $2^{n+1} e$, where $n$ is any positive integer.

As $g \in \mathcal{G}_{\theta}(\mathbb{C})$, there exists $p \in \mathbb{N}^{*}$ and $C_{p}>0$ (not depending on $n$ ) such that

$$
\max _{|\xi| \leq 2^{n+3} e}|g(\xi)| \leq C_{p} e^{\theta\left(p 2^{n}\right)} .
$$

Thus, there exists $\varepsilon_{p}>0$ (not depending on $n$ ) and $R_{n}, 2^{n} \leq R_{n} \leq 2^{n+1}$ such that

$$
\min _{|\xi|=R_{n}}|g(\xi)| \geq \varepsilon_{p} e^{-\theta\left(p 2^{n}\right)}
$$

Let $n \in \mathbb{N}$ and $|\xi|=R_{n}$. As $f \in \mathcal{G}_{\theta}(\mathbb{C})$, there exists $q>0$ and $C_{q}>0$ (not depending on $n$ ), such that

$$
|f(\xi)| \leq C_{q} e^{\theta\left(q 2^{n}\right)}
$$

Using the convexity of $\theta$ and the fact that $\theta(0)=0$, we have

$$
\theta\left(p 2^{n}\right) \leq \frac{1}{2} \theta\left(p 2^{n+1}\right)
$$

If we assume, for example, that $p \geq q$, we deduce that

$$
|h(\xi)|=|f(\xi)| \frac{1}{|g(\xi)|} \leq \frac{C_{q}}{\varepsilon_{p}} e^{\theta\left(q 2^{n}\right)+\theta\left(p 2^{n}\right)} \leq B_{p} e^{\theta\left(p 2^{n+1}\right)} .
$$

Now let $z \in \mathbb{C}$, be such that $2^{n-1} \leq|z| \leq 2^{n}<R_{n}$. By the maximum modulus theorem,

$$
|h(z)| \leq B_{p} e^{\theta\left(p 2^{n+1}\right)} \leq B_{p} e^{\theta(4 p|z|)}
$$

This proves that $h \in \mathcal{G}_{\theta}(\mathbb{C})$.

Corollary 4. In the case where $\Phi$ has no zeros, the only mean-periodic function $f \in \mathcal{F}_{\theta}(\mathbb{C})$ is the zero function. 
Proof. Assume that $\Phi$ has no zeros. Then $\frac{1}{\Phi} \in \mathcal{H}(\mathbb{C})$ and, by Lemma $3, \frac{1}{\Phi} \in \mathcal{G}_{\theta}(\mathbb{C})$. By Proposition 2, $S=(\mathcal{L})^{-1}\left(\frac{1}{\Phi}\right) \in \mathcal{F}_{\theta}^{\prime}(\mathbb{C})$. Then, we have $S \star T=T \star S=\delta_{0}$. If we assume $T \star f=0$, then $\delta_{0} \star f=f=0$.

We will throughout the rest of the paper assume that $\Phi$ has zeros, and denote them by $\left|\alpha_{0}\right| \leq$ $\left|\alpha_{1}\right| \leq \cdots \leq\left|\alpha_{k}\right| \leq \cdots, \alpha_{k} \neq \alpha_{k^{\prime}}$ if $k \neq k^{\prime}$.

We will denote by $m_{k}$ be the order of multiplicity of $\Phi$ at $\alpha_{k}$ and we will consider the "multiplicity variety" $V=\left\{\left(\alpha_{k}, m_{k}\right)\right\}_{k \in \mathbb{N}}$ (see [1] for an introduction to the concept of multiplicity variety). We may denote $V=\Phi^{-1}(0)$.

Lemma 5. (i) For all $\xi \in \mathbb{C}$ and $l \in \mathbb{N}$, we have $<T, M_{l, \xi}>=\Phi^{(l)}(\xi)$.

(ii) Each exponential monomial $M_{l, \alpha_{k}}$, for $0 \leq l<m_{k}$, is T-mean-periodic.

\section{Proof.}

To prove (i), we proceed by induction on $l \geq 0$. The property is true for $l=0$ by definition of the Fourier-Borel transform of $T$.

Suppose the property true for $l$. Let $\xi \in \mathbb{C}$ be fixed. Let us verify that the function $\frac{M_{l, \xi+u}-M_{l, \xi}}{u}$ converges in $\mathcal{F}_{\theta}(\mathbb{C})$ to $M_{l+1, \xi}$ when $u$ tends to 0 . For all $z \in \mathbb{C}$ and $u \leq 1$, we have

$$
\left|\frac{e^{u z}-1}{u}-z\right|=\left|u z^{2} \sum_{n \geq 2} \frac{(u z)^{n-2}}{n !}\right| \leq|u||z|^{2} e^{|z|} \text {. }
$$

This implies that

$$
\left|\frac{M_{l, \xi+u}(z)-M_{l, \xi}(z)}{u}-M_{l+1, \xi}(z)\right|=\left|z^{l} e^{\xi z}\right|\left|\frac{e^{u z}-1}{u}-z\right| \leq|u||z|^{l+2} e^{(1+|\xi|)|z|} .
$$

Therefore, $\frac{M_{l, \xi+u}-M_{l, \xi}}{u}$ converges to $M_{l+1, \xi}$ for the topology of $\mathcal{F}_{\theta}(\mathbb{C})$ when $u$ tends to 0 .

From this, we obtain

$$
\begin{aligned}
\Phi^{(l+1)}(\xi) & =\lim _{u \rightarrow 0} \frac{\Phi^{(l)}(\xi+u)-\Phi^{(l)}(\xi)}{u}=\lim _{u \rightarrow 0} \frac{<T, M_{l, \xi+u}>-<T, M_{l, \xi}>}{u} \\
& =\lim _{u \rightarrow 0}<T, \frac{M_{l, \xi+u}-M_{l, \xi}}{u}>=<T, M_{l+1, \xi}>,
\end{aligned}
$$

by continuity of $T$. This completes the proof of (i).

In order to prove (ii), it is sufficient to see that

$$
T \star M_{l, \alpha_{k}}(z)=<T, \tau_{z} M_{l, \alpha_{k}}>=e^{\alpha_{k} z} \sum_{n=0}^{l} C_{l}^{n} z^{l-n}<T, M_{n, \alpha_{k}}>=e^{\alpha_{k} z} \sum_{n=0}^{l} C_{l}^{n} z^{l-n} \Phi^{(n)}\left(\alpha_{k}\right) .
$$

Theorem 6. (i) Any T-mean-periodic function $f \in \mathcal{F}_{\theta}(\mathbb{C})$ admits the following expansion as a convergent series in $\mathcal{F}_{\theta}(\mathbb{C})$

$$
f(z)=\sum_{k \geq 0} \sum_{l=0}^{m_{k}-1} c_{k, l}\left[\sum_{j=0}^{k} e^{z \alpha_{j}} P_{k, j, l}(z)\right]
$$


where $P_{k, j, l}$ are the polynomials of degree $<m_{j}$ given by (23) and (24). The coefficients $c_{k, l}$ verify the following estimate

$$
\forall m>0, \quad \sum_{k \geq 0} e^{\theta\left(m\left|\alpha_{k}\right|\right)}\left(\sum_{l=0}^{m_{k}-1}\left|c_{k, l}\right|\left(\left|\alpha_{k}\right|+1\right)^{-\left(m_{1}+\cdots+m_{k-1}+l\right)}\right)<+\infty
$$

and are given by

$$
c_{k, l}=<S_{k, l}, f>
$$

where $S_{k, l} \in \mathcal{F}_{\theta}^{\prime}(\mathbb{C})$ is defined by

$$
\mathcal{L}\left(S_{k, l}\right)(\xi)=\left(\xi-\alpha_{k}\right)^{l} \prod_{n=0}^{k-1}\left(\xi-\alpha_{n}\right)^{m_{n}} .
$$

(ii) Conversely, any such serie whose coefficients $c_{n, l}$ satisfy the estimate $(10)$ converges in $\mathcal{F}_{\theta}(\mathbb{C})$ to a function $f$ solving the equation (6).

Corollary 7. Assume that all the multiplicities $m_{k}$ are equal to 1 . Then

(i) any $T$-mean-periodic function $f \in \mathcal{F}_{\theta}(\mathbb{C})$ admits the following expansion as a convergent series in $\mathcal{F}_{\theta}(\mathbb{C})$

$$
f(z)=\sum_{k \geq 0} c_{k}\left[\sum_{j=0}^{k} e^{z \alpha_{j}} \prod_{0 \leq n \leq k, n \neq j}\left(\alpha_{j}-\alpha_{n}\right)^{-1}\right],
$$

where the coefficients $c_{k}$ satisfy the following estimate

$$
\forall m>0, \quad \sum_{k \geq 0} e^{\theta\left(m\left|\alpha_{k}\right|\right)}\left|c_{k}\right|\left(\left|\alpha_{k}\right|+1\right)^{-(k-1)}<+\infty
$$

and are given by

$$
c_{k}=<S_{k}, f>
$$

where $S_{k} \in \mathcal{F}_{\theta}^{\prime}(\mathbb{C})$ is defined by

$$
\mathcal{L}\left(S_{k}\right)(\xi)=\prod_{n=0}^{k-1}\left(\xi-\alpha_{n}\right)^{m_{n}}
$$

(ii) Conversely, any such series whose coefficients $c_{k}$ satisfy the estimate $(12)$ converges in $\mathcal{F}_{\theta}(\mathbb{C})$ to a function $f$ solving the equation (6).

\section{PRoOf of TheOREM 6}

It will be a crucial point in the proof of the main theorem, to determine the image of the restriction operator $\rho$ defined on $\mathcal{G}_{\theta}(\mathbb{C})$ by

$$
\rho(g)=\left\{\frac{g^{l}\left(\alpha_{k}\right)}{l !}\right\}_{k \geq 0,0 \leq l<m_{k}}, \quad g \in \mathcal{G}_{\theta}(\mathbb{C}) .
$$

As an immediate consequence of Lemma 3, we have the following lemma.

Lemma 8. The kernel of the restriction operator $\rho$ is the ideal generated by $\Phi$ in $\left.\mathcal{G}_{\theta}(\mathbb{C})\right\}$, i.e.,

$$
\operatorname{Ker} \rho=\left\{\Phi g, g \in \mathcal{G}_{\theta}(\mathbb{C})\right\} \text {. }
$$


We are going to use a characterization, obtained in [16], of the elements $a=\left\{a_{k, l}\right\}_{k \in \mathbb{N}, 0 \leq l<m_{k-1}}$ belonging to $\rho\left(\mathcal{G}_{\theta}(\mathbb{C})\right)$. This characterization is given in terms of growth conditions involving the divided differences (see [10] for further details about divided differences).

To any discrete doubly indexed sequence $a=\left\{a_{k, l}\right\}_{k \in \mathbb{N}, 0 \leq l<m_{k}}$ of complex numbers, we associate the sequence of divided differences $\Psi(a)=\left\{b_{k, l}\right\}_{k \in \mathbb{N}, 0 \leq l<m_{k}}$. We recall that they are the coefficients of the Newton polynomials,

$$
Q_{q}(\xi)=\sum_{k=0}^{q} \prod_{n=0}^{k-1}\left(\xi-\alpha_{n}\right)^{m_{n}}\left(\sum_{l=0}^{m_{k}-1} b_{k, l}\left(\xi-\alpha_{k}\right)^{l}\right),
$$

defined, for any $q \geq 1$, as the unique polynomial of degree $m_{0}+\cdots+m_{q}-1$ such that

$$
\frac{Q_{q}^{(l)}\left(\alpha_{k}\right)}{l !}=a_{k, l}, \text { for } 0 \leq k \leq q \text { and } 0 \leq l \leq m_{k}-1 .
$$

When all the multiplicities $m_{k}=1$, we may give a simple formula for the coefficients $b_{k}$ :

$$
b_{k}=\sum_{j=0}^{k} a_{j} \prod_{0 \leq n \leq k, n \neq j}\left(\alpha_{j}-\alpha_{n}\right)^{-1} .
$$

In the general case, we define them by induction :

$$
\begin{gathered}
b_{1, l}=a_{1, l}, \text { for all } 0 \leq l \leq m_{1}-1, \\
b_{k, 0}=\frac{a_{k, 0}-Q_{k=1}\left(\alpha_{k}\right)}{\Pi_{k-1}\left(\alpha_{k}\right)}, \\
b_{k, l}=\frac{a_{k, l}-\frac{Q_{k-1}^{(l)}\left(\alpha_{k}\right)}{l !}-\sum_{n=0}^{l-1} \frac{1}{(l-n) !} \Pi_{k-1}^{(l-k)}\left(\alpha_{j}\right) b_{k, n}}{\Pi_{k-1}\left(\alpha_{k}\right)} \text { for } 1 \leq l \leq m_{k}-1
\end{gathered}
$$

where we have denoted by

$$
\Pi_{k}(\xi)=\prod_{n=0}^{k}\left(\xi-\alpha_{n}\right)^{m_{n}}, \text { for all } k \geq 0 .
$$

In [16], we showed that the elements $a=\left\{a_{k, l}\right\}_{k \in \mathbb{N}, 0 \leq l<m_{k}}$ belonging to $\rho\left(\mathcal{G}_{\theta}(\mathbb{C})\right)$ are precisely those verifying the growth condition

$$
\sup _{k \geq 0} \sup _{0 \leq l<m_{k}}\left|b_{k, l}\right|\left(\left|\alpha_{k}\right|+1\right)^{m_{1}+\cdots+m_{k-1}+l} e^{-\theta\left(m\left|\alpha_{k}\right|\right)}<+\infty,
$$

for a certain $m>0$, where $b=\left\{b_{k, l}\right\}_{k, 0 \leq l<m_{k}}=\Psi^{-1}(a)$.

In order to give a topological structure to this space, let us denote by $\mathcal{B}_{\theta, m}(V)$ the Banach space of all doubly indexed sequences of complex numbers $b=\left\{b_{k, l}\right\}_{k \in \mathbb{N}, 0 \leq l<m_{k}}$ such that

$$
\|b\|_{\theta, m}=\sup _{k \in \mathbb{N}} \sup _{0 \leq l<m_{k}}\left|b_{k, l}\right|\left(\left|\alpha_{k}\right|+1\right)^{m_{1}+\cdots+m_{k-1}+l} e^{-\theta\left(m\left|\alpha_{k}\right|\right)}<+\infty .
$$

Let us consider the space $\mathcal{A}_{\theta, m}(V)=\Psi^{-1}\left(\mathcal{B}_{\theta, m}(V)\right)$, that is, the space of all doubly indexed sequences of complex numbers $a=\left\{a_{k, l}\right\}_{k \in \mathbb{N}^{*}, 0 \leq l<m_{k}}$ such that

$$
\|\Psi(a)\|_{\theta, m}<+\infty \text {. }
$$


It is easy to see that $\mathcal{A}_{\theta, m}(V)$ endowed with the norm $\|a\|_{\theta, m}=\|\Psi(a)\|_{\theta, m}$ is a Banach space and that $\Psi$ is an isometry from $\mathcal{A}_{\theta, m}(V)$ into $\mathcal{B}_{\theta, m}(V)$.

Now, we define the spaces

$$
\mathcal{A}_{\theta}(V)=\cup_{p \in \mathbb{N}^{*}} \mathcal{A}_{\theta, p}(V) \text { and } \mathcal{B}_{\theta}(V)=\cup_{p \in N^{*}} \mathcal{B}_{\theta, p}(V)
$$

endowed with the topology of inductive limit of Banach spaces.

We define the linear map

$$
\alpha=\Psi \circ \rho \circ \mathcal{L}: \mathcal{F}_{\theta}^{\prime}(\mathbb{C}) \rightarrow \mathcal{B}_{\theta}(V) .
$$

Proposition 9. The map $\alpha$ is continuous and surjective.

Proof. By Proposition 2, we know that $\mathcal{L}: \mathcal{F}_{\theta}^{\prime}(\mathbb{C}) \rightarrow \mathcal{G}_{\theta}(\mathbb{C})$ is a topological isomorphism.

The fact that the operator

$$
\rho: \mathcal{G}_{\theta}(\mathbb{C}) \rightarrow \mathcal{A}_{\theta}(V)
$$

is continuous and surjective are easy consequences of [16. Proposition 1.8 and Theorem 1.11].

Finally, by construction, it is clear that

$$
\Psi: \mathcal{A}_{\theta}(V) \rightarrow \mathcal{B}_{\theta}(V)
$$

is a topological isomorphism.

Recall that $\mathcal{F}_{\theta}(\mathbb{C})$ is a Fréchet-Schwartz space, therefore it is reflexive. Then, the transpose $\alpha^{t}$ of $\alpha$ is defined from the strong dual of $\mathcal{B}_{\theta}(V)$, denoted by $\mathcal{B}_{\theta}^{\prime}(V)$, into $\mathcal{F}_{\theta}(\mathbb{C})$.

In the next lemma, we will characterize the dual space $\mathcal{B}_{\theta}^{\prime}(V)$.

Lemma 10. The space $\mathcal{B}_{\theta}^{\prime}(V)$ is topologically isomorphic through the canonical bilinear form

$$
<c, b>=\sum_{k=0}^{+\infty} \sum_{l=0}^{m_{k}-1} c_{k, l} b_{k, l}
$$

to the space $\mathcal{C}_{\theta}(V)=\cap_{p \in \mathbb{N}^{*}} \mathcal{C}_{\theta, p}(V)$ endowed with the projective limit topology, where, for all $p$, $\mathcal{C}_{\theta, p}(V)$ is the Banach space of the sequences $c=\left\{c_{k, l}\right\}_{k \geq 0,0 \leq l<m_{k}}$ such that

$$
\|c\|_{\theta, p}^{\prime}:=\sum_{k \geq 0} e^{\theta\left(p\left|\alpha_{k}\right|\right)}\left(\sum_{l=0}^{m_{k}-1}\left|c_{k, l}\right|\left(\left|\alpha_{k}\right|+1\right)^{-\left(m_{1}+\cdots+m_{k-1}+l\right)}\right)<+\infty .
$$

Moreover, $\mathcal{C}_{\theta}(V)$ is a Fréchet-Schwartz space.

Proof.

Let us show that $\beta: \mathcal{C}_{\theta}(V) \rightarrow \mathcal{B}_{\theta}^{\prime}(V)$ defined by $\beta(c)(b)=<c, b>=\sum_{k=0}^{+\infty} \sum_{l=0}^{m_{k}-1} c_{k, l} b_{k, l}$ is a topological isomorphism.

Let $c=\left\{c_{k, l}\right\}_{k, 0 \leq l<m_{k}}$ be an element of $\mathcal{C}_{\theta}(V)$ and $b=\left\{b_{k, l}\right\}_{k, 0 \leq l<m_{k}} \in \mathcal{B}_{\theta, p}(V)$, for a certain $p$. For any $k \geq 0$, we have, by definition of $\|b\|_{\theta, p}$,

$$
\sum_{l=0}^{m_{k}-1}\left|b_{k, l} c_{k, l}\right| \leq e^{\theta\left(p\left|\alpha_{k}\right|\right)}\|b\|_{\theta, m} \sum_{l=0}^{m_{k}-1}\left|c_{k, l}\right|\left(\left|\alpha_{k}\right|+1\right)^{-\left(m_{1}+\cdots+m_{k-1}+l\right)} .
$$

Using the estimate (14), we see that the sum converges (absolutely) and that

$$
|<c, b>| \leq\|c\|_{\theta, p}^{\prime}\|b\|_{\theta, p} \text {. }
$$


This shows the continuity of $\beta$. Let $B^{k, l}$ be the doubly indexed sequence of $\mathbb{C}$ defined by (using the Kronecker symbols) :

$$
B^{k, l}=\left\{\delta_{k j} \delta_{l n}\right\}_{j, 0 \leq n<m_{j}}
$$

We easily see that $B^{k, l} \in \mathcal{B}_{\theta, p}(V)$. For all $k$ and $0 \leq l<m_{k}$, we have $c_{k, l}=<\beta(c), B^{k, l}>$. It is then clear that $\beta$ is injective. Conversely, to an element $\nu \in \mathcal{B}_{\theta}^{\prime}(V)$, consider the doubly indexed sequence $c=\left\{c_{k, l}\right\}_{k, 0 \leq l<m_{k}}$ defined by

$$
c_{k, l}=<\nu, B^{k, l}>
$$

We verify that $c \in \mathcal{C}_{\theta}(V)$. let $p \in \mathbb{N}^{*}$ be fixed and define $\tilde{b}=\left\{\tilde{b}_{k, l}\right\}_{k, 0 \leq l<m_{k}}$ by

$$
\tilde{b}_{k, l}=e^{\theta\left(p\left|\alpha_{k}\right|\right)} \frac{\bar{c}_{k, l}}{\left|c_{k, l}\right|}\left(\left|\alpha_{k}\right|+1\right)^{-\left(m_{1}+\cdots+m_{k-1}+l\right)} \text { if } c_{k, l} \neq 0, \quad \tilde{b}_{k, l}=0 \text { otherwise. }
$$

It is clear that $\tilde{b} \in \mathcal{B}_{\theta, p}(V)$ and that $\|\tilde{b}\|_{\theta, p} \leq 1$.

Therefore, all the finite sequences $\tilde{b}^{K}=\sum_{k=0}^{K} \sum_{l=0}^{m_{k}-1} \tilde{b}_{k, l} B^{k, l}$ satisfy

$$
\left\|\tilde{b}^{K}\right\|_{\theta, p} \leq 1 \text {. }
$$

Denoting by $\|\nu\|_{p}$ the operator norm, we have, for all $K$,

$$
\left|<\nu, \tilde{b}^{K}>\right| \leq\|\nu\|_{p}\left\|\tilde{b}^{K}\right\|_{\theta, p} \leq\|\nu\| .
$$

On the other hand,

$$
<\nu, \tilde{b}^{K}>=\sum_{k=0}^{K} \sum_{l=0}^{m_{k}-1} \tilde{b}_{k, l}<\nu, B^{k, l}>=\sum_{k=0}^{K} e^{\theta\left(p\left|\alpha_{k}\right|\right)} \sum_{l=0}^{m_{k}-1}\left|c_{k, l}\right|\left(\left|\alpha_{k}\right|+1\right)^{-\left(m_{1}+\cdots+m_{k-1}+l\right)},
$$

by definition of $\tilde{b}_{k, l}$. Letting $K$ tend to infinity, we obtain that $c \in \mathcal{C}_{\theta, p}(V)$ and that

$$
\|c\|_{\theta, p} \leq\|\nu\|_{p}
$$

Consider now an element $b=\left\{b_{k, l}\right\}_{k, 0 \leq l<m_{k}}$ of $\mathcal{B}_{\theta, p}(V)$ and put

$$
b^{K}=\sum_{k=0}^{K} \sum_{l=0}^{m_{k}-1} b_{k, l} B^{k, l} .
$$

Let $q$ be an integer strictly larger than $p$. Note that by convexity of $\theta$, for all $k$ the following inequality holds

$$
-\theta\left(q\left|\alpha_{k}\right|\right)+\theta\left(p\left|\alpha_{k}\right|\right) \leq-(1-p / q) \theta\left(q\left|\alpha_{k}\right|\right)
$$

Using this inequality, we find

$$
\left\|b-b^{K}\right\|_{\theta, q} \leq\|b\|_{\theta, p} \sup _{k>K} e^{-\theta\left(q\left|\alpha_{k}\right|\right)+\theta\left(p\left|\alpha_{k}\right|\right)} \leq\|b\|_{\theta, p} e^{-(1-p / q) \theta\left(q\left|\alpha_{K}\right|\right)} .
$$

We readily deduce that $b^{K}$ converges to $b$ when $K$ tends towards infinity and that $\beta(c)=\nu$.

The continuity of $\beta^{-1}$ is a direct consequence of the inequality $(16)$. We have proved that $\beta$ is a topological isomorphism. 
In order to prove that $\mathcal{C}_{\theta}(V)$ is a Fréchet-Schwartz space, in view of [2, Proposition 1.4.8.], it is sufficient to see that, for any $p \in \mathbb{N}^{*}$, the canonical injection

$$
i_{p}: \mathcal{C}_{\theta, p+1}(V) \rightarrow \mathcal{C}_{\theta, p}(V)
$$

is compact. Let $\left\{c^{n}\right\}_{n}$ be a sequence of elements in $\mathcal{C}_{\theta, p+1}(V)$ such that, for all $n,\left\|c^{n}\right\|_{\theta, p+1} \leq 1$. It suffices to show that one can extract a subsequence of $\left\{c^{n}\right\}_{n}$ converging in $\mathcal{C}_{\theta, p}(V)$.

It is easy to see that, for all $k \in \mathbb{N}$ and $0 \leq l<m_{k}$ the sequence $\left\{c_{k, l}^{n}\right\}_{n}$ is bounded. Thus, up to taking a subsequence, we may assume without loss of generality that $c_{k, l}^{n}$ converges to a certain $c_{k, l} \in \mathbb{C}$. Putting $c=\left\{c_{k, l}\right\}_{k, 0 \leq l<m_{k}}$, we readily see that $c \in \mathcal{C}_{\theta, p+1}(V)$ and $\|c\|_{\theta, p+1} \leq 1$.

Let us verify that $\left\|c^{n}-c\right\|_{\theta, p}$ tends to 0 when $n$ tends to infinity. We assume that $\left|\alpha_{k}\right| \rightarrow \infty$, otherwise, the result is trivial. Then, again using inequality $(17)$ we find that $e^{\theta\left(p\left|\alpha_{k}\right|\right)-\theta\left((p+1)\left|\alpha_{k}\right|\right)}$ tends to 0 when $k$ tends towards infinity. Let $\varepsilon>0$. For a certain $K \in \mathbb{N}$ and for all $k \geq K$, $e^{\theta\left(p\left|\alpha_{k}\right|\right)-\theta\left((p+1)\left|\alpha_{k}\right|\right)}<\frac{\varepsilon}{4}$. Thus, for all $n \in \mathbb{N}$,

$$
\begin{array}{cc} 
& \sum_{k \geq K} e^{\theta\left(p\left|\alpha_{k}\right|\right)}\left(\sum_{l=0}^{m_{k}-1}\left|c_{k, l}^{n}-c_{k, l}\right|\left(\left|\alpha_{k}\right|+1\right)^{-\left(m_{1}+\cdots+m_{k-1}+l\right)}\right) \\
\leq \quad & \frac{\varepsilon}{4} \sum_{k \geq K} e^{\theta\left((p+1)\left|\alpha_{k}\right|\right)}\left(\sum_{l=0}^{m_{k}-1}\left|c_{k, l}^{n}-c_{k, l}\right|\left(\left|\alpha_{k}\right|+1\right)^{-\left(m_{1}+\cdots+m_{k-1}+l\right)}\right) \\
\leq \quad & \frac{\varepsilon}{4}\left\|c^{n}-c\right\|_{\theta, p+1} \leq \frac{\varepsilon}{4}\left(\left\|c^{n}\right\|_{\theta, p+1}+\|c\|_{\theta, p+1}\right) \leq \frac{\varepsilon}{2} .
\end{array}
$$

Moreover, for a certain $N \in \mathbb{N}$ and for all $n \geq N$, we have

$$
\sum_{k=0}^{K-1} e^{\theta\left(p\left|\alpha_{k}\right|\right)}\left(\sum_{l=0}^{m_{k}-1}\left|c_{k, l}^{n}-c_{k, l}\right|\left(\left|\alpha_{k}\right|+1\right)^{-\left(m_{1}+\cdots+m_{k-1}+l\right)}\right) \leq \frac{\varepsilon}{2} .
$$

Finally, for $n \geq N,\left\|c^{n}-c\right\|_{\theta, p}<\varepsilon$.

From now on, we will identify $\mathcal{B}_{\theta}^{\prime}(V)$ with the space $\mathcal{C}_{\theta}(V)$. The duality is given by, for all $c=\left\{c_{k, l}\right\}_{k, 0 \leq l<m_{k}} \in \mathcal{C}_{\theta}(V)$ and for all $b=\left\{b_{k, l}\right\}_{k, 0 \leq l<m_{k}} \in \mathcal{B}_{\theta}(V)$,

$$
<c, b>=\sum_{k=0}^{+\infty} \sum_{l=0}^{m_{k}-1} c_{k, l} b_{k, l} .
$$

The next step is to prove the following lemma :

Lemma 11. $(i) \alpha^{t}$ is a topological isomorphim onto its image and $\operatorname{Im} \alpha^{t}=(\operatorname{Ker} \alpha)^{\circ}$, the orthogonal space of $\operatorname{Ker} \alpha$.

(ii) $\operatorname{Ker} \alpha=\left\{T \star U, U \in \mathcal{F}_{\theta}^{\prime}(\mathbb{C})\right\}$.

(iii) $(\operatorname{Ker} \alpha)^{\circ}=\operatorname{Ker} T \star=\left\{f \in \mathcal{F}_{\theta}(\mathbb{C}) \mid T \star f=0\right\}$.

\section{Proof.}

(i) From Proposition 9, $\alpha$ is a surjective continuous linear map. Therefore, $\alpha^{t}$ is a topological isomorphism onto its image and $\operatorname{Im} \alpha^{t}=(\operatorname{Ker} \alpha)^{\circ}($ see [2, Proposition 1.4.12]).

(ii) Recalling Remark 8, we have

$$
\left.\operatorname{Ker} \alpha=\operatorname{Ker}(\rho \circ \mathcal{L})=\mathcal{L}^{-1}(\operatorname{Ker} \rho)=\left\{T \star \mathcal{L}^{-1}(g), g \in \mathcal{G}_{\theta}(\mathbb{C})\right\}\right)=\left\{T \star U, \quad U \in \mathcal{F}_{\theta}^{\prime}(\mathbb{C})\right\} .
$$


(iii) Let $f$ be an element of $(\operatorname{Ker} \alpha)^{\circ}$. For all $z \in \mathbb{C}$,

$$
(T \star f)(z)=<T, \tau_{z} f>=<T, \delta_{z} \star f>=<T \star \delta_{z}, f>=0,
$$

using the fact that $T \star \delta_{z} \in \operatorname{Ker} \alpha$.

Conversely, let $f \in \mathcal{F}_{\theta}(\mathbb{C})$ be such that $T \star f=0$ and let $U \in \mathcal{F}_{\theta}^{\prime}(\mathbb{C})$. We have

$$
<T \star U, f>=<U, T \star f>=0 .
$$

This shows that $f \in(\operatorname{Ker} \alpha)^{\circ}$ and concludes the proof of the lemma.

Let us proceed with the proof of Theorem 6 .

(i) Let $f \in \mathcal{F}_{\theta}(\mathbb{C})$ be a $T$-mean-periodic function, that is, $f \in \operatorname{Ker} T \star$. From Lemmas 11 and 10 , there is a unique sequence $c \in \mathcal{C}_{\theta}(V)$ such that $f=\alpha^{t}(c)$.

For $z \in \mathbb{C}$, denoting by $\delta_{z}$ the Dirac measure at $z$, we have

$$
f(z)=<\delta_{z}, f>=<\delta_{z}, \alpha^{t}(c)>=<c, \alpha\left(\delta_{z}\right)>=<c, \Psi\left(\rho\left(g_{z}\right)\right)>
$$

where we have denoted by $g_{z}=\mathcal{L}\left(\delta_{z}\right)$, that is, the function in $\mathcal{G}_{\theta}(\mathbb{C})$ defined by $g_{z}(\xi)=e^{z \xi}$.

Let us compute $\Psi\left(\rho\left(g_{z}\right)\right)=b(z)=\left\{b_{k, l}(z)\right\}_{k, 0 \leq l<m_{k}}$, which is an element of $\mathcal{B}_{\theta}(V)$. By well known formulas about Newton polynomials (See, for example [2, Definition 6.2.8]), we have, for $k \in \mathbb{N}$, and denoting by

$$
\partial_{j}^{m}=\frac{1}{m !} \frac{\partial^{m}}{\partial \alpha_{j}^{m}}
$$

for $0 \leq l<m_{k}$,

$$
b_{k, l}(z)=\partial_{1}^{m_{1}-1} \cdots \partial_{k-1}^{m_{k-1}-1} \partial_{k}^{l}\left(\sum_{j=0}^{k} e^{z \alpha_{j}} \prod_{0 \leq n \leq k, n \neq j}\left(\alpha_{j}-\alpha_{n}\right)^{-1}\right)=\sum_{j=0}^{k} e^{z \alpha_{j}} P_{k, j, l}(z),
$$

where we have denoted by

$$
P_{k, j, l}(z)=\sum_{i=0}^{m_{k}-1} \frac{z^{i}}{i !} \partial_{i}^{m_{j}-1-i}\left(\prod_{0 \leq n \leq k-1, n \neq j}\left(\alpha_{j}-\alpha_{n}\right)^{-m_{n}}\left(\alpha_{j}-\alpha_{k}\right)^{-(l+1)}\right)
$$

and, for $0 \leq l<m_{k}$,

$$
P_{k, k, l}(z)=\sum_{i=0}^{l} \frac{z^{i}}{i !} \partial_{k}^{l-i}\left(\prod_{0 \leq n \leq k-1}\left(\alpha_{k}-\alpha_{n}\right)^{-m_{n}}\right) .
$$

Thus,

$$
f(z)=\sum_{k \geq 0}\left(\sum_{l=0}^{m_{k}-1} c_{k, l} b_{k, l}(z)\right)=\sum_{k \geq 0}\left(\sum_{l=0}^{m_{k}-1} c_{k, l} \sum_{j=0}^{k} e^{z \alpha_{j}} P_{k, j, l}(z)\right) .
$$

So, the equality (11) is established.

Let us verify the convergence in $\mathcal{F}_{\theta}(\mathbb{C})$ of the series.

Case where $\theta(x)=x$. Here, $\mathcal{F}_{\theta}(\mathbb{C})=\mathcal{H}(\mathbb{C})$. We have to verify that the serie converges uniformly on every compact of $\mathbb{C}$. Let $p \in \mathbb{N}^{*}$ and $z \in \mathbb{C},|z| \leq p$.

We have, for all $\xi \in \mathbb{C},\left|g_{z}(\xi)\right|=\left|e^{z \xi}\right| \leq e^{p|\xi|}$, that is,

$$
\left\|g_{z}\right\|_{\theta, p} \leq 1
$$


Thus, by continuity of $\Psi \circ \rho$, there exists $p^{\prime} \in \mathbb{N}^{*}$ and $C_{p}>0$ such that

$$
\|b(z)\|_{\theta, p^{\prime}} \leq C_{p}\left\|g_{z}\right\|_{\theta, p} \leq C_{p}
$$

For all $k \geq 0$, we have

$$
\left|\sum_{l=0}^{m_{k}-1} c_{k, l} b_{k, l}(z)\right| \leq\|b(z)\|_{\theta, p^{\prime}} \sum_{l=0}^{m_{k}-1}\left|c_{k, l}\right|\left(\left|\alpha_{k}\right|+1\right)^{-\left(m_{1}+\cdots+m_{k-1}+l\right)} e^{\theta\left(p^{\prime}\left|\alpha_{n}\right|\right)} .
$$

We obtain

$$
\sup _{|z| \leq p}\left|\sum_{l=0}^{m_{k}-1} c_{k, l} b_{k, l}(z)\right| \leq C_{p} \sum_{l=0}^{m_{k}-1}\left|c_{k, l}\right|\left(\left|\alpha_{k}\right|+1\right)^{-\left(m_{1}+\cdots+m_{k-1}+l\right)} e^{\theta\left(p^{\prime}\left|\alpha_{k}\right|\right)}
$$

Recalling that $c \in \mathcal{C}_{\theta}^{\prime}(V)$, the right term is the general term of a convergent serie, thus, the right-hand side of $(11)$ is convergent in $\mathcal{F}_{\theta}(\mathbb{C})$. Moreover,

$$
\sup _{|z| \leq p} \sum_{k \geq 0}\left|\sum_{l=0}^{m_{k}-1} c_{k, l} b_{k, l}(z)\right| \leq C_{p}\|c\|_{\theta, p^{\prime}}^{\prime}
$$

Case where $\theta$ is a Young function.

For any $p \in \mathbb{N}^{*}$, observe that

$$
\left\|g_{z}\right\|_{\theta, p} \leq e^{\theta^{*}\left(\frac{1}{p}|z|\right)}
$$

Thus, by continuity of $\Psi \circ \rho$, there exists $p^{\prime} \in \mathbb{N}^{*}$ and $C_{p}>0$ such that

$$
\|b(z)\|_{\theta, p^{\prime}} \leq C_{p}\left\|g_{z}\right\|_{p} \leq C_{p} e^{\theta^{*}\left(\frac{1}{p}|z|\right)} .
$$

For all $k \geq 0$ and $z \in \mathbb{C}$, we have

$$
\left|\sum_{l=0}^{m_{k}-1} c_{k, l} b_{k, l}(z)\right| \leq\|b(z)\|_{\theta, p^{\prime}} \sum_{l=0}^{m_{k}-1}\left|c_{k, l}\right|\left(\left|\alpha_{k}\right|+1\right)^{m_{1}+\cdots+m_{k-1}+l} e^{-\theta\left(p^{\prime}\left|\alpha_{k}\right|\right)} .
$$

We obtain

$$
\sup _{z \in \mathbb{C}}\left|\sum_{l=0}^{m_{k}-1} c_{k, l} b_{k, l}(z)\right| e^{-\theta^{*}\left(\frac{1}{p}|z|\right)} \leq C_{p} \sum_{l=0}^{m_{k}-1}\left|c_{k, l}\right|\left(\left|\alpha_{k}\right|+1\right)^{m_{1}+\cdots+m_{k-1}+l} e^{-\theta\left(p^{\prime}\left|\alpha_{k}\right|\right)} .
$$

As in the previous case, we deduce that the right-hand side of (11) is absolutely convergent in $\mathcal{F}_{\theta}(\mathbb{C})$. Moreover,

$$
\sup _{z \in \mathbb{C}} \sum_{k \geq 0}\left|\sum_{l=0}^{m_{k}-1} c_{k, l} b_{k, l}(z)\right| \leq C_{p}\|c\|_{\theta, p^{\prime}}^{\prime}
$$

In order to find an explicit formula for the coefficients $c_{n, l}$, consider the elements $B^{k, l}$ of $\mathcal{B}_{\theta}(\mathbb{C})$ defined by (15) and observe that, by the definition of the Newton polynomials (see (13) ) with respect to the coefficients of $B^{k, l}$, for all $q \geq k$, we have

$$
Q_{q}(\xi)=\left(\xi-\alpha_{k}\right)^{l} \prod_{l=0}^{k-1}\left(\xi-\alpha_{l}\right)^{m_{l}}
$$

and for $q<k, Q_{q}=0$. We readily deduce that $\alpha\left(S_{k, l}\right)=\Psi \circ \rho \circ \mathcal{L}\left(S_{k, l}\right)=B^{k, l}$. 
Now, for all $k \in \mathbb{N}$ and $0 \leq l<m_{k}$,

$$
<S_{k, l}, f>=<S_{k, l}, \alpha^{t}(c)>=<\alpha\left(S_{k, l}\right), c>=<B^{k, l}, c>=c_{k, l} .
$$

(ii) The converse part is easily deduced from the proof of (i) and Lemma 5 .

\section{CASE WHERE $V$ IS AN INTERPOLATING VARIETY.}

Definition. We say that $V$ is an interpolating variety for $\mathcal{G}_{\theta}(\mathbb{C})$ if, for any doubly indexed sequence $a=\left\{a_{k, l}\right\}_{k \in \mathbb{N}^{*}, 0 \leq l<m_{k}}$ such that, for a certain $m>0$,

$$
\sup _{k \in \mathbb{N}} \sum_{l=0}^{m_{k}-1}\left|a_{k, l}\right| e^{-\theta\left(\left.m\right|_{k} \mid\right)}<+\infty,
$$

there exists a function $g \in \mathcal{G}_{\theta}(\mathbb{C})$ such that, for all $k$ and all $0 \leq l<m_{k}-1$,

$$
\frac{g^{(l)}\left(\alpha_{k}\right)}{l !}=a_{k, l} .
$$

We assume from now on that $V$ is an interpolating variety for $\mathcal{G}_{\theta}(\mathbb{C})$. Then we have the following result :

Theorem 12. (i) Any T-mean-periodic function $f \in \mathcal{F}_{\theta}(\mathbb{C})$ admits the following expansion as a convergent series in $\mathcal{F}_{\theta}(\mathbb{C})$

$$
f(z)=\sum_{k \geq 0} e^{z \alpha_{k}} \sum_{l=0}^{m_{k}-1} d_{k, l} \frac{z^{l}}{l !},
$$

where the coefficients $a_{k, l}$ verify the following estimate :

$$
\sum_{k \geq 0} e^{\theta\left(m\left|\alpha_{k}\right|\right)}\left(\sum_{l=0}^{m_{k}-1}\left|d_{k, l}\right|\right)<+\infty
$$

for every $m>0$. Moreover, for all $k \in \mathbb{N}$ and $0 \leq l<m_{k}$, we have the equality

$$
d_{k, l}=<T_{k, l}, f>
$$

where $T_{k, l} \in \mathcal{F}_{\theta}^{\prime}(\mathbb{C})$ is defined by

$$
\mathcal{L}\left(T_{k, l}\right)(\xi)=\Phi(\xi)\left(\xi-\alpha_{k}\right)^{l-m_{k}}\left(m_{k} ! / \Phi^{\left(m_{k}\right)}\left(\alpha_{k}\right)\right),
$$

(ii) Conversely, any such series whose coefficients $d_{k, l}$ satisfy the estimate (26) converges in $\mathcal{F}_{\theta}(\mathbb{C})$ to a function $f$ solving the equation (6).

Note that $\mathcal{L}\left(T_{k, l}\right) \in \mathcal{G}_{\theta}(\mathbb{C})$ by Proposition 3 .

Remark 13. In the case where $\theta(x)=x$, this is also a consequence of [2, Theorem 6.2.6.].

We will denote by $\mathcal{A}_{\theta, m}(V)$ the space of all doubly indexed sequences of complex numbers $a=\left\{a_{k, l}\right\}_{k \in \mathbb{N}, 0 \leq l<m_{k}}$ such that

$$
\|a\|_{\theta, m}:=\sup _{k \in \mathbb{N}} \sum_{l=0}^{m_{k}-1}\left|a_{k, l}\right| e^{-\theta\left(m\left|\alpha_{k}\right|\right)}<+\infty
$$


and

$$
\mathcal{A}_{\theta}(V)=\cup_{p \in N^{*}} \mathcal{A}_{\theta, p}(V)
$$

endowed with the strict inductive limit of Banach spaces.

We define the linear map

$$
\alpha=\rho \circ \mathcal{L}: \mathcal{F}_{\theta}^{\prime}(\mathbb{C}) \rightarrow \mathcal{A}_{\theta}(V) .
$$

Proposition 14. The map $\alpha$ is continuous and surjective.

Proof.

It is sufficient to show that the map $\rho: \mathcal{G}_{\theta}(\mathbb{C}) \rightarrow \mathcal{A}_{\theta}(V)$ is continous and surjective. The surjectivity follows from the fact that $V$ is an interpolating variety.

In order to show the continuity, let $g \in \mathcal{G}_{\theta, p}(\mathbb{C})$ and let $z \in \mathbb{C}$. By the Cauchy estimates applied to the disc of center $z$ and radius 2 , for all $l \in \mathbb{N}$,

$$
\left|\frac{g^{(l)}(z)}{l !}\right| \leq \frac{1}{2^{l}} \sup _{|\xi-z| \leq 2}|g(\xi)| \text {. }
$$

For $|\xi-z| \leq 2$, we have

$$
|g(\xi)| \leq\|g\|_{\theta, p} e^{\theta(p|\xi|)} \leq\|g\|_{\theta, p} e^{\theta(2 p+p|z|)} \leq\|g\|_{\theta, p} e^{1 / 2 \theta(4 p)} e^{1 / 2 \theta(2 p|z|)}
$$

by convexity of $\theta$. Thus,

$$
\sum_{l=0}^{\infty}\left|\frac{g^{l}(z)}{l !}\right| \leq 2\|g\|_{\theta, p} e^{1 / 2 \theta(2 p)} e^{\theta(2 p|z|)} .
$$

In particular, we deduce that $\rho(g) \in \mathcal{A}_{\theta, 2 p}(V)$ and that

$$
\|\rho(g)\|_{\theta, 2 p} \leq 2\|g\|_{\theta, p} e^{1 / 2 \theta(2 p)} .
$$

The continuity of $\rho$ follows from the last inequality and the surjectivity from the definition 5 .

We need to characterize $\mathcal{A}_{\theta}^{\prime}(V)$, the strong dual of $\mathcal{A}_{\theta}(V)$.

Lemma 15. The space $\mathcal{A}_{\theta}^{\prime}(V)$ is topologically isomorphic to the space $\mathcal{D}_{\theta}(V)=\cap_{p \in \mathbb{N}^{*}} \mathcal{C}_{\theta, p}(V)$ endowed with the projective limit topology, where, for all $p, \mathcal{D}_{\theta, p}(V)$ is the Banach space of the sequences $d=\left\{d_{k, l}\right\}_{k, 0 \leq l<m_{k}}$ such that

$$
\|d\|_{\theta, p}^{\prime}:=\sum_{k \geq 0} e^{\theta\left(p\left|\alpha_{k}\right|\right)}\left(\sum_{l=0}^{m_{k}-1}\left|d_{k, l}\right|\left(\left|\alpha_{k}\right|+1\right)^{-\left(m_{1}+\cdots+m_{k-1}+l\right)}\right)<+\infty .
$$

Moreover, $\mathcal{D}_{\theta}(V)$ is a Fréchet-Schwartz space.

The proof is similar to the one of Lemma 10 . Lemma 8 still holds with the new definition of $\alpha$. We are now ready to prove Theorem 12 .

By Lemma 8 , any $T$-mean-periodic function $f$ is the image by $\alpha^{t}$ of a unique $d \in \mathcal{A}_{\theta}^{\prime}(V)$. We have, for all $z \in \mathbb{C}$,

$$
f(z)=<\delta_{z}, f>=<\delta_{z}, \alpha^{t}(d)>=<d, \alpha\left(\delta_{z}\right)>=<d, \rho\left(g_{z}\right)>=\sum_{k \geq 0} e^{z \alpha_{k}} \sum_{l=0}^{m_{k}-1} \frac{z^{l}}{l !} d_{k, l} .
$$


To compute the coefficients $d_{k, l}$ :

$$
<T_{k, l}, f>=<T_{k, l}, \alpha^{t}(d)>=<d, \alpha\left(T_{k, l}\right)>=d_{k, l} .
$$

The last equality follows from the observation that

$$
\alpha\left(T_{k, l}\right)=B^{k, l} .
$$

The rest of the proof is similar to the one of Theorem 6 .

Let us recall some results about interpolating varieties that enables one to determine whether $V$ is interpolating or not. The following is an analytic characterization given in [2, Chapter 2]. The spaces of entire functions considered are slightly different, but is clear how to adapt these results to our spaces.

Theorem 16. Assume $V=\Phi^{-1}(0)$. Then $V$ is an interpolating variety for $\mathcal{G}_{\theta}(\mathbb{C})$ if and only if, there are constants $\varepsilon>0$ and $m>0$ such that, for all $k$ and all $0 \leq l<m_{k}$,

$$
\left|\frac{\Phi^{\left(m_{k}\right)}(z)}{l !}\right| \geq \varepsilon e^{-\theta\left(m\left|\alpha_{k}\right|\right)} .
$$

We also have a geometric characterization of interpolating varieties, in terms of the distribution of the points $\left\{\left(\alpha_{k}, m_{k}\right)\right\}_{k}$.

Define the counting function and the integrated counting function :

Definition. For $z \in \mathbb{C}$ and $r>0$,

$$
\begin{gathered}
n(z, r)=\sum_{\left|z-\alpha_{k}\right| \leq r} m_{k}, \\
N(z, r)=\int_{0}^{r} \frac{n(z, t)-n(z, 0)}{t} d t+n(z, 0) \ln r=\sum_{0<\left|z-\alpha_{k}\right| \leq r} m_{k} \ln \frac{r}{\left|z-\alpha_{k}\right|}+n(z, 0) \ln r .
\end{gathered}
$$

Adapting [3, Corollary 4.8] or [15, Theorem 2.9] to our spaces :

Theorem 17. $V$ is an interpolating variety for $\mathcal{G}_{\theta}(\mathbb{C})$ if and only if conditions

$$
\exists A>0, \exists m>0 \quad \forall R>0, \quad N(0, R) \leq A+\theta(m R)
$$

and

$$
\exists A>0, \exists m>0 \quad \forall k \in \mathbb{N}, \quad N\left(\alpha_{k},\left|\alpha_{k}\right|\right) \leq A+\theta\left(m\left|\alpha_{k}\right|\right)
$$

hold.

Actually, in this paper, since $V=\Phi^{-1}(0)$ and $\Phi \in \mathcal{G}_{\theta}(\mathbb{C})$, condition 28 is necessarily verified (see [16, Theorem 1.13]). Thus, $V$ is an interpolating variety if and only if condition (29) holds. 
Remark 18. We can obtain Theorem 12 as a corollary of Theorem 6, using the density condition (29). This second proof is rather technical, we will skip it here. Let us just give the correspondence between the coefficients $c_{k, l}$ and $d_{k, l}$ :

$$
\begin{aligned}
d_{k, l} & =\sum_{i=l}^{m_{k}-1} c_{k, i} \partial_{k}^{i-l}\left(\prod_{0 \leq n \leq k-1}\left(\alpha_{k}-\alpha_{n}\right)^{-m_{n}}\right) \\
& +\sum_{j=k+1}^{\infty} \sum_{i=0}^{m_{j}-1} c_{j, i} \partial_{k}^{m_{k}-1-l}\left(\prod_{0 \leq n \leq j-1, n \neq k}\left(\alpha_{k}-\alpha_{n}\right)^{-m_{n}}\left(\alpha_{k}-\alpha_{j}\right)^{-(i+1)}\right),
\end{aligned}
$$

the convergence of the second sum being a consequence of conditions (29) and (28). In the case where all $m_{k}=1$, we have

$$
d_{k}=\sum_{j=k}^{\infty} c_{j} \prod_{0 \leq n \leq j, n \neq k}\left(\alpha_{k}-\alpha_{n}\right)^{-1} .
$$

\section{REFERENCES}

[1] C. A. Berenstein and R. Gay. Complex variables, volume 125 of Graduate Texts in Mathematics. SpringerVerlag, New York, (1991). An introduction.

[2] C. A. Berenstein and R. Gay. Complex analysis and special topics in harmonic analysis. Springer-Verlag, New York, 1995.

[3] C. A. Berenstein and B. Q. Li. Interpolating varieties for spaces of meromorphic functions. J. Geom. Anal., 5 (1995), 1-48.

[4] C. A. Berenstein and D. C. Struppa. Dirichlet series and convolution equations Publ. RIMS, Kyoto Univ., 24 (1988), 783-810.

[5] C. A. Berenstein and D. C. Struppa. Complex analysis and convolution equations. Itogi Nauki Tekh., Ser. Sovrem. Probl. Mat., Fundam. Napravleniya. 54 (1989) 5-111; English transl. Several complex variables. V: Complex analysis in partial differential equations and mathematical physics, Encycl. Math. Sci., 54 (1993) $1-108$.

[6] C. A. Berenstein and B. A. Taylor. A new look at interpolation theory for entire functions of one variable. Adv. in Math., 33 (1979), 109-143.

[7] J. Delsarte. Les fonctions moyennes-périodiques. J. Math. Pures et Appl., 14 (1935), 403-453.

[8] J. Dieudonné and L. Schwartz. La dualité dans les espaces $(\mathcal{F})$ et $(\mathcal{L F})$. Annales de l'institut Fourier, 1 (1949), $1-14$.

[9] R. Gannoun, R. Hachaichi, H. Ouerdiane, and A. Rezgui. Un théorème de dualité entre espaces de fonctions holomorphes à croissance exponentielle. J. Funct. Anal., 171 (2000), 1-14.

[10] E. Isaacson and H. B Keller. Analysis of numerical methods. Dover Publications Inc., New York, 1994. Corrected reprint of the 1966 original [Wiley, New York].

[11] Krasičkov, I. F. Closed ideals in a locally convex algebra of entire functions with an arbitrary majorant of growth. Dokl. Akad. Nauk SSSR, 170 (1966), 1018-1019.

[12] Krasičkov, I. F. Closed ideals in locally convex algebras of entire functions. Algebras of minimal type. Sibirsk. Mat. Z., 9 (1968), 77-96.

[13] Krasičkov, I. F. Closed ideals in locally convex algebras of entire functions. II. Izv. Akad. Nauk SSSR Ser. Mat., 32 (1968), 1024-1032.

[14] M. A. Krasnosel'skii and Ya. B. Ritickii. Convex Functions and Orlicz Spaces, P. Noordhoff. Itd, Groningen, 1961. 
[15] M. Ounaïes. Geometric Conditions for Interpolation in Weighted Spaces of Entire Functions. J. Geom. Anal., 17 (2008), 701-716.

[16] M. Ounaïes. Interpolation by entire functions with growth conditions. Michigan Math. J., 56 (2008), 155-171.

[17] L. Schwartz. Espaces nucléaires. Seminaire Schwartz, 1 (Exposé 17), 1935.

[18] L. Schwartz. Théorie générale des fonctions moyenne-périodiques. Ann. Math., 48 (4) (1947), 857-929.

Departement de mathématiques, Université Tunis-El Manar, Tunisie

Institut de Recherche Mathématique Avancée, Université de Strasbourg, 7 Rue René DesCARTES, 67084 Strasbourg CEDEX, France. 